FRANCHFRI: the Finite-RANge Constrained Hartree-Fock Rapid Iterator

W. Younes, D. Gogny

February 1, 2007 
This document was prepared as an account of work sponsored by an agency of the United States Government. Neither the United States Government nor the University of California nor any of their employees, makes any warranty, express or implied, or assumes any legal liability or responsibility for the accuracy, completeness, or usefulness of any information, apparatus, product, or process disclosed, or represents that its use would not infringe privately owned rights. Reference herein to any specific commercial product, process, or service by trade name, trademark, manufacturer, or otherwise, does not necessarily constitute or imply its endorsement, recommendation, or favoring by the United States Government or the University of California. The views and opinions of authors expressed herein do not necessarily state or reflect those of the United States Government or the University of California, and shall not be used for advertising or product endorsement purposes.

This work was performed under the auspices of the U.S. Department of Energy by University of California, Lawrence Livermore National Laboratory under Contract W-7405-Eng-48. 


\title{
FRANCHFRI: the Finite-RANge Constrained Hartree-Fock Rapid Iterator
}

\author{
W. Younes and D. Gogny \\ Lawrence Livermore National Laboratory, Livermore, California 94551
}

(Dated: 30th January 2007)

\begin{abstract}
The Hartree-Fock code FRANCHFRI, which uses a finite-range nucleon-nucleon interaction, has been written and benchmarked. This code represents a new LLNL capability for realistic calculations in both nuclear-structure and nuclear-reaction physics. The use of a finite-range interaction represents a considerable improvement over other Hartree-Fock codes currently available in the public domain, which rely on zero-range forces. The finite-range force does not simply lead to a more realistic treatment of the nuclear problem, it avoids serious mathematical pathologies inherent to zero-range interactions. This brief and non-technical report introduces the code, its design philosophy, various benchmarks used to test its accuracy, and places it within its proper physics context. The current limitations and planned extensions of the code are also discussed.
\end{abstract}

\section{INTRODUCTION}

The complete and accurate description of nuclei is a many-body problem, encompassing phenomena from the single-particle behavior of a few valence nucleons to the collective motion of many or all the constituent nucleons. The nuclear theorist's arcanum, therefore, is a tractable solution to this general many-body problem. Unfortunately, while two-body systems can still be described analytically, the general many-body problem is not amenable to an exact mathematical treatment, and must be tackled by some approximation. The HartreeFock (HF) method provides an approximate solution to the many-body problem with two important features: i) for many applications, it constitutes a very good first approximation, and ii) there are well-established, rigorous procedures for improving the HF solution and restoring the physics missing in the approximation.

In the HF approximation, the many-body wave function of the nucleus is reduced to a single Slater determinant of single-particle wave functions. The exact nuclear wave function is a linear superposition of an infinite number of such Slater determinants. A variational procedure is used to select the Slater determinant that minimizes the energy of the system. This variational procedure can be written as a set of non-linear equations that must be solved iteratively. Another approximation inherent to the HF method is the neglect of residual interactions. Instead, each nucleon interacts with a mean field generated by all the nucleons. The HF method in its most basic incarnation, therefore, is well-adapted to the description of the ground state of magic nuclei, for which the excitation of nucleons to valence orbitals is energetically costly. However, even these basic calculations can give surprisingly good predictions for the ground-state properties of nuclei far from magicity, as shown in Fig. 1.

The input to the HF procedure is the effective (i.e., inmedium) interaction between the nucleons, used to generate the mean field. The mathematical form of this interaction is constrained, but not completely and uniquely defined by symmetry requirements (e.g., rotational and translational invariance). Therefore an explicit form must be postulated for this interaction, and its parameters must be fixed by a fit to experimental observables. The general form for the HF interaction consists of a central part, a spin-orbit interaction, a density-dependent contribution, and a Coulomb interaction which is applied only between protons. The density-dependent part of the force is motivated by more fundamental approaches, such as the Brueckner G-matrix theory, which describes the nucleon-nucleon interaction in the presence of other nucleons, using an approach that is formally similar to that of scattering theory.

The earliest form used for the central part of the interaction was introduced by T. H. R. Skyrme [1], and consisted of a delta function with its second-order derivatives. The main appeal of this force is its relative mathematical simplicity, but although it is still used in modern calculations, it does not take into account the finite range of the nuclear interaction. In fact, the Skyrme interaction suffers from insidious mathematical pathologies [2] which prevent any self-consistent extension of the HF method to include the residual interactions beyond the mean-field approximation. The limitations of the Skyrme interaction are most easily grasped in a Fourier analysis of the zero-range force. Then, the delta function in the relative nucleon-nucleon coordinates, and its second-order partials, transform into functions which are constant and quadratic in the relative momenta, respectively. Instead of vanishing rapidly with high relative momenta, as in the case of finite-range forces, the constant term yields the perplexing result that a pair of nucleons can be imparted any relative momentum from 0 to $\infty$ with equal probability. Worse yet, the quadratic term preferentially scatters the nucleon pair to ever-higher relative momenta. These pathological behaviors are hidden to some extent for bound states by the localized form of their wave functions, but cannot be avoided when considering particlehole excitations of the nucleus, whether for the study of excited states or to restore missing correlations in the description of ground-state properties. When considering these properties, proponents of the Skyrme interac- 


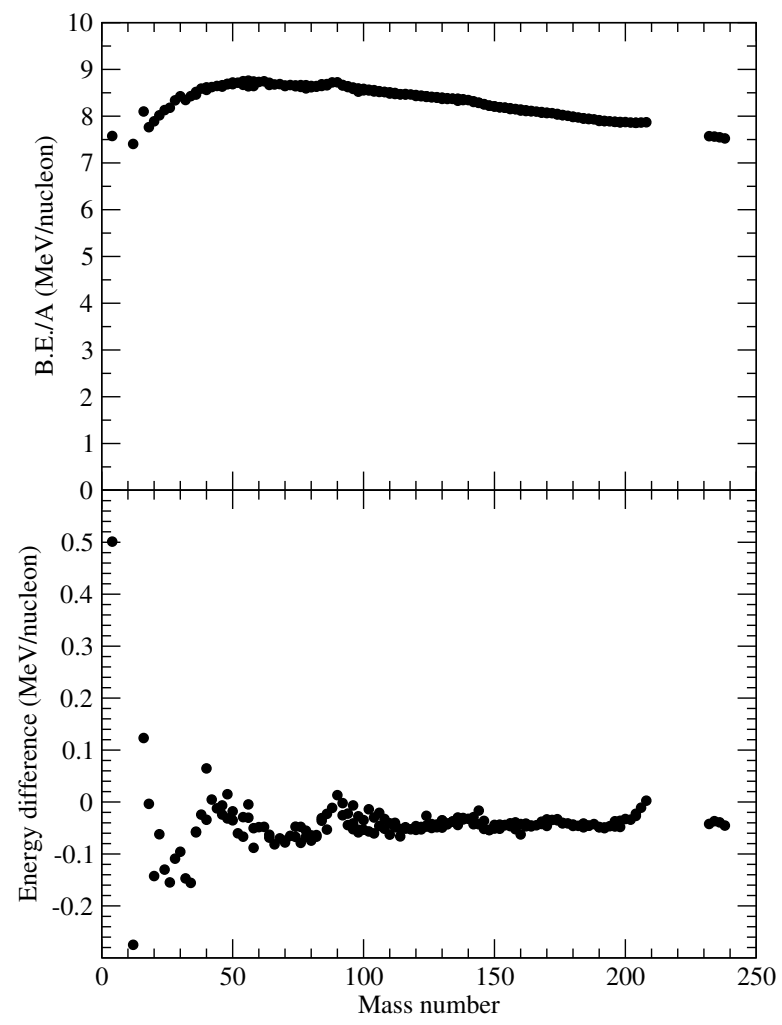

Figure 1: Top panel: binding energies per nucleon calculated for stable and long-lived even-even nuclei using the HartreeFock code FRANCHFRI with the Gogny D1S force. Bottom panel: corresponding difference between calculated and measured binding energies per nucleon. The discrepancies between calculated and measured values are mainly due to correlations missing in a pure HF calculation.

tion are forced to introduce ad-hoc procedures to placate the inherent pathologies of the delta functions, and in the process, they sacrifice the central tenet of the HF methodology: self-consistency. In 1975, D. Gogny introduced a finite-range form of the central part of the force [3], as an alternative to the Skyrme interaction. The D1S parameterization of the Gogny force, used in the calculations in Fig. 1, was fixed by a fit to properties of nuclear matter and of the nuclei ${ }^{16} \mathrm{O},{ }^{90} \mathrm{Zr}$, and ${ }^{208} \mathrm{~Pb}$.

The approximations inherent to the HF method make it a tractable approach to the nuclear many-body problem. For some applications, however, a more sophisticated treatment may be required, and the physics omitted in the HF approximation, mainly in the form of missing residual correlations, must be restored. Moving away from magic nuclei, the experimentally-observed decrease in the energy gap for even-even nuclei signals an increase in the importance of pair correlations between nucleons, relative to the mean-field approximation. For these nuclei, the residual pair correlations are usually included using the prescription given by Bogoliubov [4], and resulting in the extension to the HF formalism known as the Hartree-Fock-Bogoliubov (HFB) method. The prescription incorporates pair correlations while maintaining, to a large extent, the simplicity of the mean-field approximation through a mathematical transformation that maps pairwise-interacting particle into non-interacting quasiparticles. This procedure does not introduce any new parameters to the HF method, but its relative simplicity is bought at the cost of violating the strict conservation of the number of nucleons. Fortunately, this broken symmetry is partially restored by imposing conservation of the average number of nucleons.

Next, the HFB procedure can be extended to describe collective motion of the nucleus. Small-amplitude motions (e.g., low-energy vibrations of the nuclear surface) give rise to particle-hole excitations across the Fermi surface, and the resulting residual interactions between particles and holes (as well as those particle-particle and hole-hole correlations beyond pairing) can be taken into account by the Quasiparticle Random-Phase Approximation (QRPA) [5]. For large-amplitude collective motion that can occur in phenomena such as shape isomerism, fusion, and fission, the nucleus can explore shapes far from that given by the HFB solution. The HFB method is designed to find a single particular shape of the nucleus, the one that minimizes its energy, but can readily be extended by introducing external fields to yield solutions for any desired nuclear shape. The Constrained Hartree-Fock-Bogoliubov (CHFB) method introduces the required external fields via Lagrange multipliers. Each CHFB solution is a single Slater determinant for a given nuclear shape, but a large-amplitude collective motion of the nucleus consists of a mixture of all these solutions. The Generator-Coordinate Method (GCM) constructs such a linear superposition of the CHFB solutions through a variational procedure.

In summary, the HF method can be viewed as a starting point for the solution of the nuclear many-body problem. The extensions listed above can then be used to describe a very wide array of phenomena in nuclear structure and nuclear reactions. The HF method with all its extensions is a powerful, versatile tool which requires an effective nucleon-nucleon interaction as its only input.

\section{IMPLEMENTATION}

Some of the extensions described above (HFB, CHFB) can be built directly into the $\mathrm{HF}$ procedure, while the others (QRPA, GCM) are applied to the HFB solutions a-posteriori. In the version of the code FRANCHFRI described in this paper, the HF method is extended to allow external constraints. With this extension, and the 
realistic finite-range Gogny interaction, a variety of nuclear shapes and their properties can be investigated. The choice of a finite-range force instead of the simpler zero-range delta interaction poses a computational challenge. In principle, two-body matrix elements of the finite-range interaction require the accurate evaluation of 6-dimensional integrals in spatial coordinates, at great computational cost. In practice, a separation technique developed by D. Gogny [6], expands the two-body matrix elements in the deformed harmonic-oscillator basis into a finite sum of products of one-body matrix elements, which can be written in closed form and evaluated very quickly. This separation method is not an approximation, but a mathematical identity that casts the two-body matrix elements into a form well-suited to fast computations. In addition, intermediate quantities that are combined to form the two-body matrix elements are calculated once and for all when the code begins execution, and need not be re-evaluated during each HF iteration, thereby leading to a substantial speed-up of the code. Two assumed symmetries of the Hamiltonian are hardwired into the current version of FRANCHFRI: time-reversal symmetry, and axial symmetry. Further symmetries, such as parity and z-axis signature, can be imposed at runtime by the user. These symmetries reduce the HF Hamiltonian matrix to block-diagonal form, thereby decreasing the number of non-zero two-body matrix elements that must be calculated, and further speeding up the program.

The HF calculations must be performed in the intrinsic frame of the system, which requires a subtraction of the spurious motion of the center of mass of the nucleus. This subtraction is routinely carried out to first order in many HF codes, by removing the one-body contribution of the center-of-mass motion. In the code FRANCHFRI, the full contribution of the center-of-mass motion, which includes two-body components, can optionally be subtracted out, leading to more accurate HF calculations, especially for lighter nuclei. The exchange part of the Coulomb interaction is also often calculated in an approximate way in other HF codes, using the so-called Slater approximation [7], for efficiency reasons. In FRANCHFRI, the separation method is applied to the Coulomb interaction, and the Coulomb-exchange contribution can be calculated exactly at each HF iteration or optionally, at the last iteration only for greater speed.

We close the discussion of the code with some benchmarks comparing FRANCHFRI calculations with calculations using the HF code HFBJINABE [8], which is meant for use in spherical nuclei only, and with published results for axially-symmetric systems [9]. Figure 2 shows a comparison of HF energies calculated using the codes FRANCHFRI and HFBJINABE. A more stringent test of the accuracy of the code FRANCHFRI is displayed in Fig. 3, which compares single-particle energies calculated with the two codes. To test the accu-

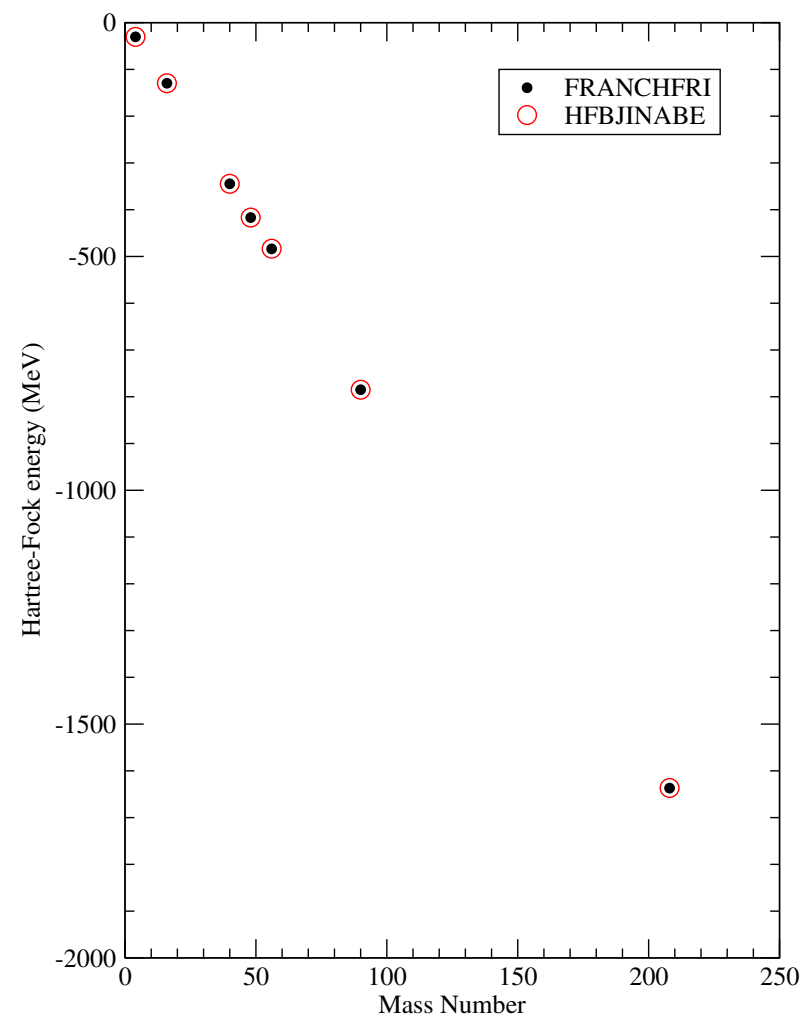

Figure 2: Comparison of HF energies calculated by the codes FRANCHFRI and HFBJINABE for the spherical nuclei ${ }^{4} \mathrm{He}$, ${ }^{16} \mathrm{O},{ }^{40} \mathrm{Ca},{ }^{48} \mathrm{Ca},{ }^{90} \mathrm{Zr}$, and ${ }^{208} \mathrm{~Pb}$.

racy of the code FRANCHFRI for non-spherical nuclei, a comparison with results in [9] of calculated HF energies is shown in Fig. 4, and the corresponding charge quadrupole moments are compared in Fig. 5. In all cases, the code FRANCHFRI reproduces the benchmark results precisely, with any remaining discrepancies attributable to negligible differences in numerical accuracy. Finally, we show in Fig. 6 the typical execution times achieved by FRANCHFRI, running on a single Intel Pentium M $2.13 \mathrm{Ghz}$ processor, for various sizes of the harmonicoscillator basis. For typical applications, therefore, the code produces results within very reasonable execution times.

\section{FUTURE DIRECTIONS}

The version of the code FRANCHFRI presented in this paper is a powerful tool, but as discussed in the introduction, its full potential once augmented by standard physics extensions, is truly formidable. One such exten- 


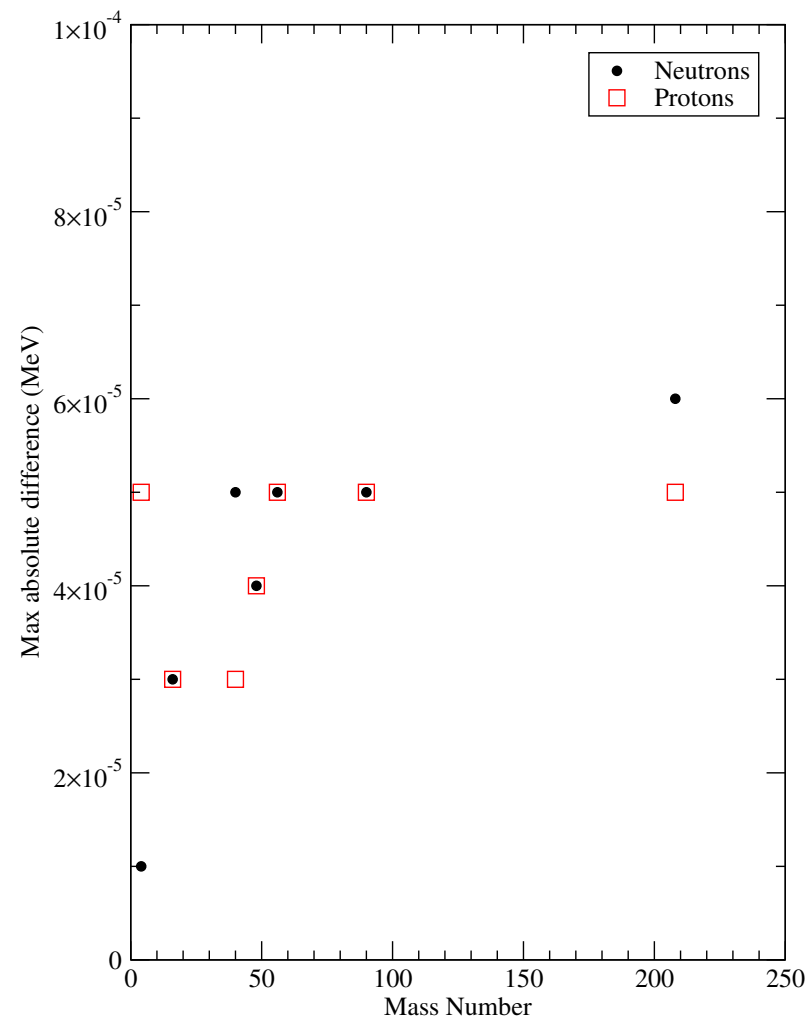

Figure 3: Largest absolute difference between neutron and proton single-particle energies of occupied states, calculated by the codes FRANCHFRI and HFBJINABE for the nuclei displayed in Fig. 2, using the full Gogny D1S interaction. Note the range of the y-axis scale, indicating that the largest discrepancy between the codes is $60 \mathrm{eV}$ for the neutron levels in ${ }^{208} \mathrm{~Pb}$. The observed discrepancies are due principally to differences in numerical accuracy, and the different number of decimals places printed by the two codes in the output files.

sion, the ability to impose external constraints on the HF solution has already been implemented.

In this section, we outline an incremental development of the code to increase its capabilities while producing, at each stage, self-contained tools that can be immediately applied to nuclear physics problems of interest. The next planned improvement to the code is the inclusion of pairing (i.e., residual particle-particle and hole-hole correlations), making it possible to perform realistic calculations in nuclei far from magicity. Once the HFB code has been implemented and tested, the axial symmetry built into the current version of FRANCHFRI can be broken, opening the door to the study of triaxial nuclei, and triaxial shapes of nuclei, such as those typically encountered during the fission process of actinides near the first barrier. Fast execution speed is an essential feature of the HF

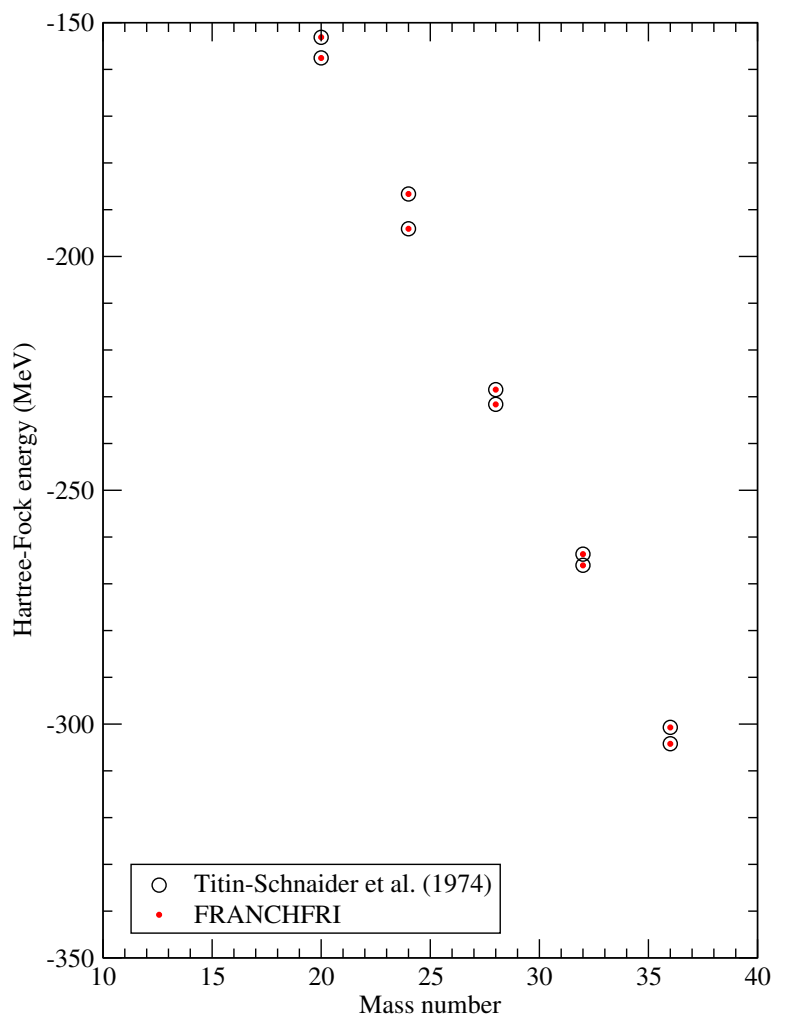

Figure 4: HF energies for the oblate and prolate minima in ${ }^{20} \mathrm{Ne},{ }^{24} \mathrm{Mg},{ }^{28} \mathrm{Si},{ }^{32} \mathrm{~S}$, and ${ }^{36} \mathrm{Ar}$, calculated using the code FRANCHFRI, and compared to calculations published in [9].

and HFB implementations, and parallel versions of the code will be developed to ensure timely calculations in large-scale applications. This will be especially useful for fission calculations, which must explore multidimensional energy surfaces requiring HF solutions for a large number of nuclear shapes. The GCM (and its time-dependent variant, the TDGCM) will be implemented in a separate code to study the dynamical aspects of large-amplitude nuclear phenomena like fission. The QRPA could also be implemented in a separate program. Of course, there are many more extensions to the basic HF formalism that can be considered, beyond the ones discussed here. Other applications that combine the HF method with shell-model [10] and shell-model Monte-Carlo [11] formalisms to restore missing correlations and perform realistic calculations of nuclear properties, look promising. Furthermore, even more realistic formulations of the Gogny force can be investigated, and a temperature-dependent $\mathrm{HF}$ formalism can be implemented to perform calculations at even higher excitation energies than is currently envisaged. 


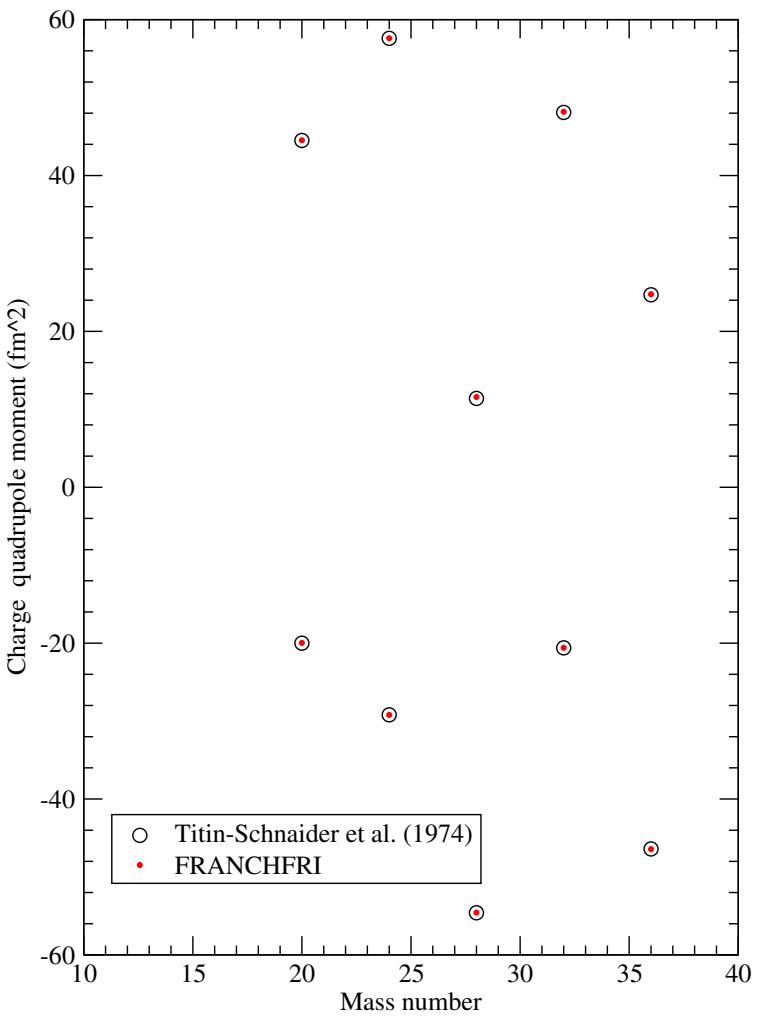

Figure 5: Comparison of charge quadrupole moments for the oblate and prolate minima in Fig. 4, between the code FRANCHFRI and the results published in [9].

From a practical point of view, the central appeal of the HF method is that its only input is the effective nucleonnucleon interaction, which is adjusted once and for all. All additional required physics is introduced in a selfconsistent way through the strict application of the laws of quantum mechanics. Therefore, it should come as no surprise that the HF method, along with its extensions has been used in almost every aspect of nuclear physics. From the prediction of energy spectra and their decay properties, to the calculation of nuclear-reaction observables, and even to the fission process, arguably the most complex and dynamic phenomenon in nuclear physics. The code FRANCHFRI discussed here, along with its planned extensions, presents LLNL with a timely opportunity to tackle a variety of nuclear-physics using a tool that implements a realistic in-medium nucleon-nucleon interaction in a code that is both fast and versatile.

This work was performed under the auspices of the U.S. Department of Energy by the University of Cali- fornia, Lawrence Livermore National Laboratory under Contract W-7405-Eng-48.

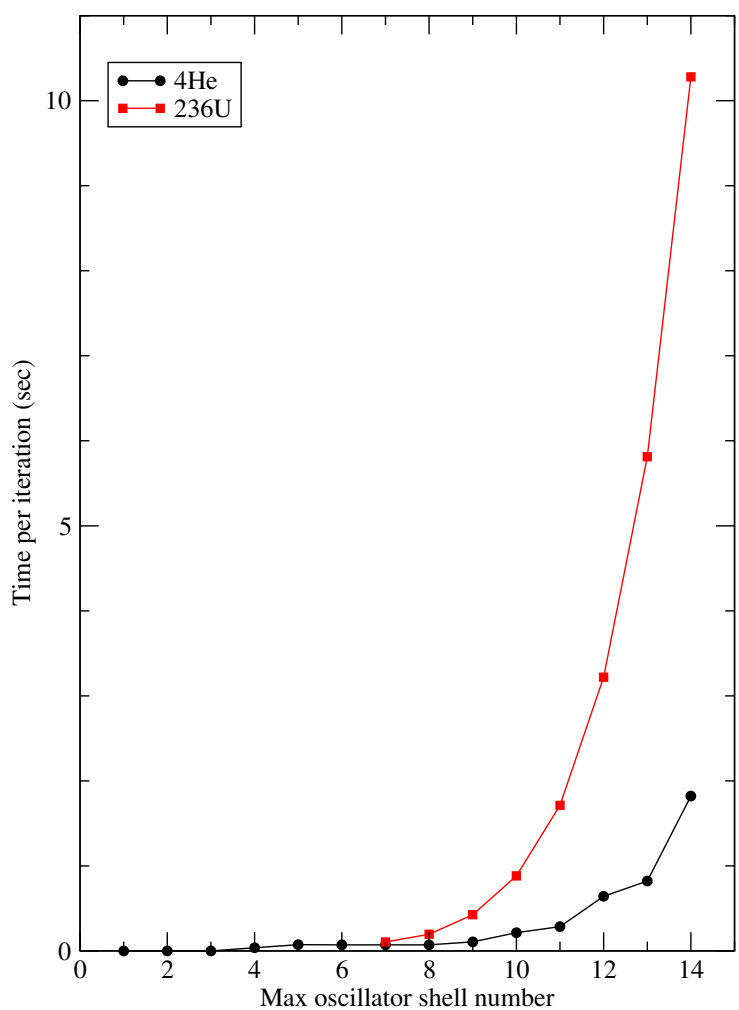

Figure 6: Execution times per $\mathrm{HF}$ iteration for ${ }^{4} \mathrm{He}$ and ${ }^{236} \mathrm{U}$ using the code FRANCHFRI, as a function of the maximum harmonic-oscillator shell number used in the basis.

[1] T. H. R. Skyrme, Phil. Mag. 1, 1043 (1956).

[2] J. Decharge and D. Gogny, Phys. Rev. C 21, 1568 (1980).

[3] D. Gogny, Proceedings of the International Conference on Nuclear Selfconsistent Fields, Trieste, (1975).

[4] N. N. Bogoliubov, Sov. Phys. JETP 7, 41 (1958).

[5] J. P. Blaizot and D. Gogny, Nucl. Phys. A284, 429 (1977).

[6] D. Gogny, Nucl. Phys. A237, 399 (1975).

[7] J. C. Slater, Phys. Rev. 81, 385 (1951).

[8] The code HFBJINABE was written by D. Gogny.

[9] C. Titin-schnaider and Ph. Quentin, Phys. Lett. B 49, 213 (1974).

[10] Y. Alhassid, G. F. Bertsch, L. Fang, and B. Sabbey, Phys. Rev. C 74, 034301 (2006).

[11] W. E. Ormand and G. Stoitcheva, private communications (2006). 